\title{
Impact of the refractive index on coupling structures for silicon solar cells
}

\author{
Laura Stevens, ${ }^{\mathrm{a}, *}$ Oliver Höhn, ${ }^{\mathrm{a}}$ Mario Hanser, ${ }^{\mathrm{a}}$ Nico Tucher, ${ }^{\mathrm{a}}$ \\ Claas Müller, ${ }^{\mathrm{b}}$ Stefan Glunz, ${ }^{\mathrm{a}, \mathrm{c}}$ and Benedikt Bläsi $\odot^{\mathrm{a}}$ \\ ${ }^{a}$ Fraunhofer Institute for Solar Energy Systems ISE, Freiburg, Germany \\ ${ }^{b}$ University of Freiburg, Department of Microsystems Engineering IMTEK, Freiburg, Germany \\ ${ }^{c}$ University of Freiburg, Department of Sustainable Systems Engineering INATECH, \\ Freiburg, Germany
}

\begin{abstract}
Structured surfaces are used to reduce reflection and enhance light-trapping in silicon solar cells. In this simulation study, we investigated the relationship between the refractive index of front-side coupling structures on top of planar wafer-based crystalline silicon solar cells and the light-trapping performance of the structures. A crossed diffraction grating with a period of $1 \mu \mathrm{m}$ and random pyramid structures with varying refractive indices were considered. Simulations were carried out both at the cell level and at the complete module stack level. It is shown that the single pass light path enhancement factor (LPEF) only provides a rough estimate of the light-trapping properties. The light-trapping behavior can only be reliably assessed in the complete system level and these results deviate from the estimated single pass LPEF. It can also be shown that the refractive index of the structure strongly influences the light-trapping behavior. (C) The Authors. Published by SPIE under a Creative Commons Attribution 4.0 Unported License. Distribution or reproduction of this work in whole or in part requires full attribution of the original publication, including its DOI. [DOI: 10.1117/1.JPE.11.027001]
\end{abstract}

Keywords: coupling structures; front side structures; light path enhancement; light-trapping; silicon solar cells.

Paper 20091 received Nov. 10, 2020; accepted for publication Mar. 15, 2021; published online Mar. 30, 2021.

\section{Introduction}

The enhancement of light absorption in wafer-based crystalline silicon ( $\mathrm{Si}$ ) solar cells can be achieved by reducing the reflection and by increasing the light-trapping in the solar cell. The state-of-the-art technique for reducing the reflection and induce light-trapping in solar cells involves a combination of a coupling structure with an antireflection coating on the front side of the device. ${ }^{1}$ These coupling structures are mostly realized by wet chemical etching, which results in random pyramids on monocrystalline silicon solar cells ${ }^{2}$ and in the so-called isotexture $^{3}$ on multicrystalline silicon solar cells. The etching process leads to an increased surface area, which then results in increased surface recombination losses.

An alternative to etching a coupling structure into silicon is the patterning of another material on top of the planar wafer. An optically structured but electrical planar front side of a solar cell has advantages such as less recombination losses, while achieving nearly the same optical performance. There are publications, which indicate light-trapping effects on the application a scattering layer of polydimethylsiloxane (PDMS) on the planar front side of a silicon and perovskite solar cell ${ }^{4,5}$ or a PDMS pyramidal structure on top of the planar front side of a III-V/ silicon solar cell. ${ }^{6}$ These concepts are mostly investigated for tandem solar cells. Using a high-index material for pillar-like coupling structures on top of a planar silicon substrate was among others presented by Spinelli et al. ${ }^{7,8}$

Studies on pillar-like coupling structures made out of $\mathrm{TiO}_{2}$-sol-gel material on top of a planar silicon wafer ${ }^{8,9}$ showed that within the investigated range of refractive index, no significant lighttrapping was achieved.

*Address all correspondence to Laura Stevens, laura.stevens@ise.fraunhofer.de 
In this paper, we focus on the influence of the refractive index of the structured layer on the light-trapping behavior of wafer-based crystalline silicon solar cells and modules. The influence of refractive index variations on light trapping has also been investigated in other contexts such as wave guides. ${ }^{10}$ For our study, the refractive index of the structured layer is varied between the refractive index of the surrounding medium ( $n=1$ in the cell case and $n=1.5$ in the module case) and the refractive index of the active cell $(n=3.5)$. In the investigated case, only the infrared wavelength range ( 1 to $1.2 \mu \mathrm{m}$ ) is relevant, as the chosen silicon solar cells with thicknesses of $\sim 200 \mu \mathrm{m}$ show imperfect absorption in this range and require light path enhancement. It is noteworthy that the thickness $200 \mu \mathrm{m}$ is chosen as a typical thickness for high efficiency solar cells. But the qualitative behavior holds also for other thickness in the wavelength range, where light-trapping is relevant. In addition, the transmittance into the system is normalized, to separate the influence of reflection and light path enhancement on the current. With these simplifications, it is possible to study the effect of refractive index alone on the light-trapping behavior. However, this parameter study does not claim to be an optimization regarding overall cell performance, which would be possible without the above-mentioned simplifications. However, in this case, the separation of the light path enhancement could not be achieved easily anymore. Three exemplary coupling structures on top of a silicon solar cell are investigated: (1) a random pyramid structure, (2) a diffraction grating structure, and (3) a Lambertian scatterer. These structures are representative of ray-optically or wave-optically working structures and one system that can be described analytically. Initially, the single pass light path enhancement factor (LPEF) was calculated using the rigorous coupled wave analysis (RCWA) ${ }^{11}$ for the grating structure and SunSolve from PV Lighthouse $^{12}$ for the random pyramid structure and the Lambertian scatterer. Subsequently, the complete system was modeled with the help of the optical properties of textured optical sheets (OPTOS) formalism ${ }^{13,14}$ and SunSolve, ${ }^{12}$ resulting in the calculation of the system LPEF and photo current enhancement. All investigations were performed at the cell and module stack level.

The paper is structured as follows: after the introduction of the simulation methods and key parameters in Sec. 2, the results for the single pass LPEF are presented in Sec. 3. In Sec. 4, the light-trapping behavior in the systems is analyzed.

\section{Methods}

Light-trapping leads to longer light paths in the substrate due to the redirection of light at different angles. An equivalent resulting absorptance can be obtained by having a thicker substrate. This equivalent larger thickness defines the LPEF. At single pass level, the light path enhancement occurs only due to the redirection of light into non-normal angles. At the system level, reflection at the backside of the substrate and light-trapping on the front side is included, which extends the light path further. This would result in an extremely thick substrate. For the description of the silicon material in all simulations the dataset from Green et al. ${ }^{15}$ was used.

\subsection{Coupling Structure Modeling}

In this simulation study, two different coupling structures were modeled: a random pyramid structure and a binary crossed grating structure. The investigations were performed for the cell case with half-infinite air at the front interface and the encapsulated case. The encapsulated case is represented by a front side embedded in a half-infinite space with $n=1.5$, as a simplified representation of the absorption free ethylene vinyl acetate (EVA) $\left(n_{\mathrm{EVA}}=1.5\right)$ and the cover glass $\left(n_{\text {Glas }}=1.5\right)$. As the refractive indices of the EVA encapsulation and the front glass are assumed to be equal, they are modeled as one layer called EVA here. The coupling structure material is assumed to be non-absorbing.

A sketch of the random pyramid structure is shown in Fig. 1(a). The pyramids have an angle of $54.7 \mathrm{deg}$. Figure 1(b) shows the periodic grating. In Fig. 1(c), a sketch of a unit cell of the square grating structure with the used geometrical parameters is shown.

The area filling factor, an additionally used parameter for the periodic crossed grating, is calculated by dividing the pillar area by the area of the unit cell ${ }^{9}$ 


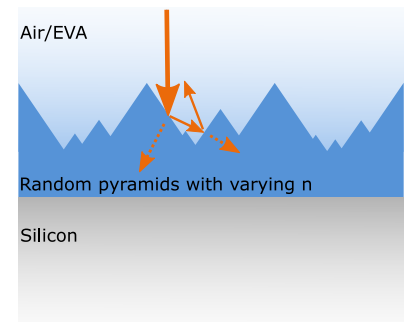

(a)

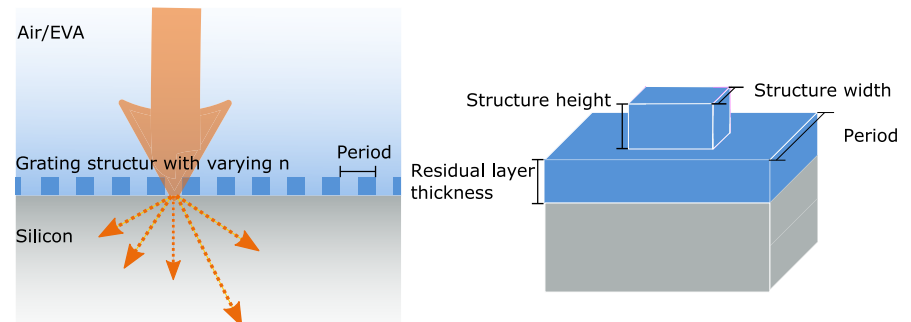

(b) (c)

Fig. 1 Sketch of the simulated coupling structures, (a) the random pyramid structure and (b) the periodic grating structure. Both systems were simulated in air and with an EVA encapsulation. ${ }^{8,9}$ (c) A sketch of the grating structure with the used parameters.

$$
\text { Filling factor }=\frac{A_{\text {structure }}}{A_{\text {unit cell }}}=\frac{\text { structure } \text { width }^{2}}{\text { period }^{2}} .
$$

\subsection{Diffraction Grating Structure-RCWA}

The RCWA method ${ }^{11}$ was used to determine the single pass LPEF for the grating. RCWA is a rigorous wave optical simulation tool that calculates the diffraction efficiencies for the diffraction angles defined by the grating equation, for a given angle of incidence. ${ }^{11,16}$

\subsection{Random Pyramid Structure-Raytracing}

The single pass and system LPEF of the random pyramid structure were modeled with the ray tracer of SunSolve from PV Lighthouse. For each component included, the optical losses and gains can be quantified individually. ${ }^{12}$

\subsection{Lambertian Scatterer-Analytically}

The Lambertian scatterer is described using diffusely scattered light according to Lambert's cosine law. The radiant intensity is proportional to the product of the cosine of the polar angle and the area.

$$
I(\theta) \sim A \cos (\theta),
$$

where $\boldsymbol{\theta}$ is the angle measured from the surface normal, and $\boldsymbol{A}$ is the area of the Lambertian surface element. Therefore, a Lambertian scatterer is placed in the material on top of the solar cell and only scatters forward. This scatterer is used as a reference system, because it is known for its excellent light-trapping and redistribution properties.

\subsection{System Modeling with OPTOS/PV-Lighthouse}

To take the multiple passes through the absorber and multiple interactions with the interfaces into account, and to rate the light-trapping and light path enhancement features more realistically, the complete system needs to be assessed. Therefore, simulations were performed using OPTOS ${ }^{13,14}$ and PV Lighthouse SunSolve. ${ }^{12}$ The modeled system consists of a structured layer with varying refractive index on top of a $200-\mu \mathrm{m}$ thick $\mathrm{Si}$ substrate with a perfect backside mirror. The coupling structures have no antireflection coating.

Figure 2 shows the investigated systems. All systems consist of a coupling structure with varying refractive index on a $\mathrm{Si}$ substrate with a perfect backside mirror $(R=1)$. The system with the EVA represents a module stack, in which an additional interface between air and EVA is introduced. For the Lambertian scatterer the front and the interlayer interface are assumed to have ideal transmission $\left(T_{\text {ideal }}\right)$. The ideal transmission is dependent on the angle of incidence as $\boldsymbol{T}_{\text {ideal }}\left(\boldsymbol{\theta}<\boldsymbol{\theta}_{\boldsymbol{c}}\right)=\mathbf{1}$ or $\boldsymbol{T}_{\text {ideal }}\left(\boldsymbol{\theta} \geq \boldsymbol{\theta}_{\boldsymbol{c}}\right)=\mathbf{0}$, where $\boldsymbol{\theta}_{\boldsymbol{c}}$ is the critical angle. 


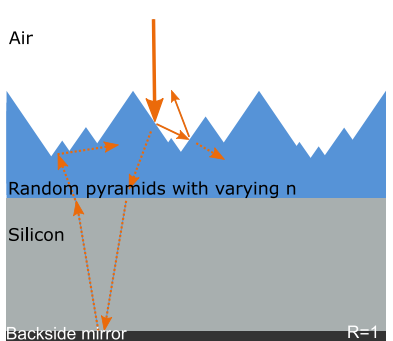

(a)

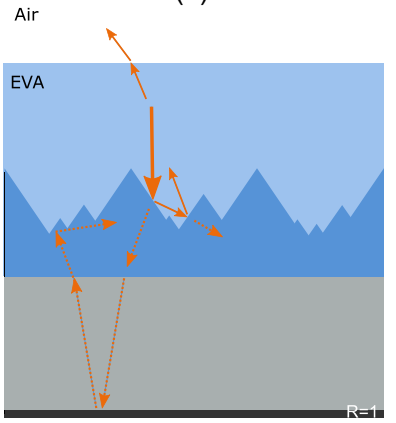

(d)

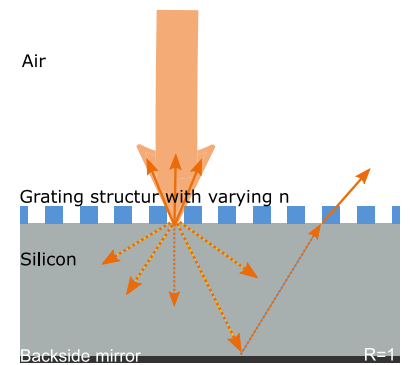

(b)

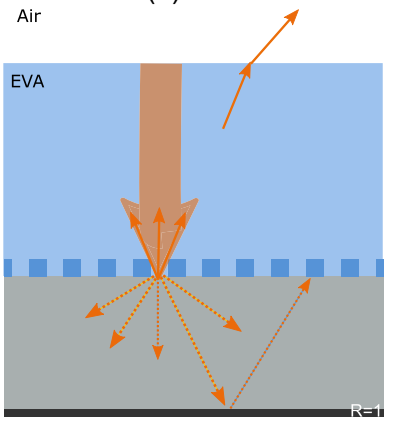

(e)

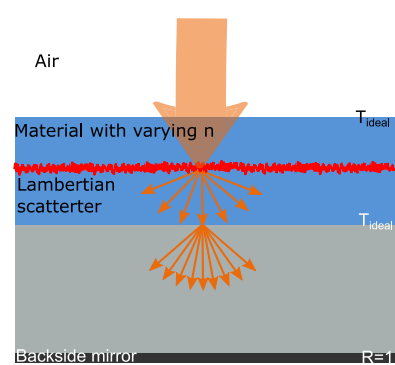

(c)

Air

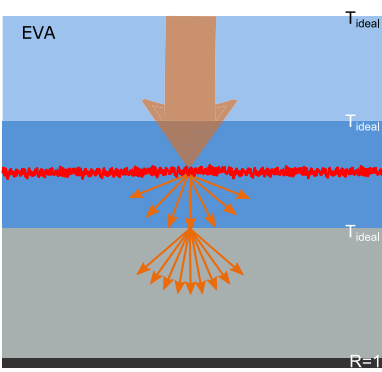

(f)

Fig. 2 Sketches of the complete simulated systems, (a) the random pyramid case in air; (b) the crossed grating in air; and (c) the Lambertian scatterer. In the lower row (d) presents the encapsulated random pyramid structure; (e) the encapsulated crossed grating; and (f) the encapsulated Lambertian scatterer. All coupling structures are on a $200-\mu \mathrm{m}$ thick Si substrate and have a perfect backside mirror $(R=1)$. For the Lambertian scatterer the front and the interlayer interface are assumed to have ideal transmission ( $\left.T_{\text {ideal }}\right)$.

In all the systems, it is possible for the light to escape at the front interface. In the cell system, this interface is between the coupling structure and air. In the module stack, this interface is between EVA and air. It is noteworthy that the real reflectance including the possibility of total internal reflection and multiple passes, was considered in this case. In addition, by assuming ideal transmission at the front and the interlayer interfaces of the Lambertian scatterer, both the cell case and the module stack obtained the same results.

\subsection{Single Pass LPEF}

The single pass LPEF was calculated for the random pyramid structure and the periodic crossed grating with a period of $1000 \mathrm{~nm}$ (1000-nm grating) with varying refractive index, placed on top of a planar silicon substrate (see Fig. 1). For the 1000-nm grating, the single pass LPEF was averaged for wavelengths between $1000 \mathrm{~nm}$ and $1150 \mathrm{~nm}$ in $50-\mathrm{nm}$ steps as the diffraction orders; thus, the single pass LPEF strongly depends on the wavelength. In this wavelength range, light-trapping in a silicon solar cell is relevant.

Light path enhancement is defined as the extension of the light path at a polar angle $\boldsymbol{\theta}_{\boldsymbol{p}}$ compared to the direct light path. Summing over all angles and weighting with the corresponding diffraction efficiency $\boldsymbol{\eta}_{\boldsymbol{p}}$ yields the single pass LPEF (formula from Ref. 17).

$$
\mathrm{LPEF}_{\text {single pass }}=\frac{\sum_{p} \beta\left(\theta_{p}\right) \eta_{p}}{\sum_{p} \eta_{p}} \quad \text { with } \quad \beta\left(\theta_{p}\right)=\frac{1}{\cos \theta_{p}} .
$$

For the random pyramid structure, the calculation of the single pass LPEF was performed using SunSolve for a fixed wavelength of $1100 \mathrm{~nm}$. As the pyramids are modeled with ray optics, the weak dependence on the wavelength is only caused by the dispersion in silicon; thus, calculating for one wavelength is sufficient. The single pass absorptance was extracted from the single pass absorptance results. A detailed explanation of how this was performed can be found in the appendix. 
The single pass LPEF for the Lambertian scatterer was calculated for a fixed silicon refractive index of 3.5. The in-coupling of the light into the Si substrate is free from reflection and the angular range of the transmitted light is limited by refraction. This leads to the following equation to calculate the single pass LPEF for the Lambertian scatterer:

$$
\mathrm{LPEF}_{\text {Lambertian scatterer }}=\frac{\int_{0}^{2 \pi} \mathrm{d} \phi \int_{0}^{\theta} \frac{\sin \theta \cos \theta}{\cos \theta} \mathrm{d} \theta}{\int_{0}^{2 \pi} \mathrm{d} \phi \int_{0}^{\theta} \sin \theta \cos \theta \mathrm{d} \theta}=\frac{1-\cos \theta}{0.5 \sin ^{2} \theta},
$$

where $\theta$ is the largest propagating angle ( $\sin \theta=n / n_{S i}$ ) in the substrate and is calculated from the Snell's law.

\subsection{System LPEF}

The system LPEF was calculated to focus on the light-trapping properties. All calculations for the system LPEF were performed at a wavelength of $1100 \mathrm{~nm}$. This wavelength was chosen because the largest absorptance enhancement caused by light-trapping for a Si thickness of $200 \mu \mathrm{m}$ is expected to be around $1100 \mathrm{~nm}$. Averaging over different wavelengths was not performed for the grating because the entire absorptance spectra showed a smooth behavior. The absorptance of the system is normalized to correct for reflection differences. This leads to the decoupling of the reflection losses and losses due to imperfect light-trapping. The normalized absorptance is calculated as

$$
\text { Absorptance }_{\text {normalized }}=\frac{\text { absorptance }}{1-\text { reflectance }_{\text {front }}} .
$$

With the help of this normalization [Eq. (5)], the LPEF for the system can be calculated without artefacts from the non-zero reflectance of the incident light on the front side (reflectance front) of the solar cell. This corresponds to the normalization (denominator) in the single pass LPEF in Eq. (3).

The OPTOS formalism ${ }^{13,14}$ was used for the 1000-nm grating. OPTOS provides the calculation of the optical properties of a system with different interfaces. Every interface is described by a redistribution matrix, which describes how the incoming light is redistributed at various angles (in reflection and transmission). Every homogeneous layer is described by a propagation matrix. This describes how light propagates and is absorbed in the medium. Using iterative matrix multiplication, the absorption in the layer can be calculated by taking multiple reflections into account.

The calculation of the system LPEF for the random pyramid structure was again performed with SunSolve.

The final system LPEF is calculated by comparing the system absorptance to the single pass Lambert-Beer-absorption. The substrate thickness needed to achieve the modeled absorptance at normal incidence is calculated $\left(d_{\text {virtual }}\right)$ and divided by the real substrate thickness $\left(d_{\text {substrate }}\right)$ of the system:

$$
\mathrm{LPEF}_{\text {system }}=\frac{d_{\text {virtual }}}{d_{\text {substrate }}}=\left(\frac{-\lambda \ln \left(1-\text { Absorptance }_{\text {normalized }}\right)}{4 \pi k}\right) / d_{\text {substrate }},
$$

Here, $\lambda$ is the wavelength, absorptance normalized is the normalized absorptance [Eq. (5)], $4 \pi k / \lambda$ represents the absorption coefficient of silicon at a specific wavelength, and $d_{\text {substrate }}$ is the thickness of the substrate (here, $200 \mu \mathrm{m}$ ).

The system LPEF for the Lambertian scatterer was also calculated by SunSolve. A Lambertian scatterer is placed in a material, the refractive index of which varied. All the interfaces in this case have perfect transmission, and the backside of the substrate is a perfect mirror.

\subsection{Calculation of the Current Density}

Finally, to be able to rate differences in the light-trapping behavior and the system LPEF at the cell level, the current density gain is modeled for the different coupling structures as compared 
to a planar substrate and compared to the differences in system LPEF. A simulation of the system was performed in the wavelength range from $700 \mathrm{~nm}$ to $1200 \mathrm{~nm}$, and the normalized absorptance in the systems was weighted with the number of photons in the AM1.5g spectrum ${ }^{18}$ and multiplied by the electric charge.

\section{Results: Single Pass LPEF}

For the presented results the methods described in Sec. 2.6 were used. The 1000-nm grating has a wider parameter range than the pyramidal structure. Preliminary simulations showed that the single pass LPEF is slightly better without a residual layer. Therefore, in all subsequent simulations, the residual layer thickness was set to 0 . The coupling structure height was varied between 0 and $1 \mu \mathrm{m}$ and the filling factor between 0 and 1 . The results of the variation can be found in the appendix.

The maximal LPEF by varying the parameter range was determined for different refractive indices of the1000-nm grating. For the 1000-nm grating structure in air, the diffraction efficiencies into the different diffraction angles were also calculated. These results are visualized in Fig. 3, which shows the influence of the structure refractive index on the diffraction efficiencies. It can easily be seen that an increasing refractive index leads to higher diffraction efficiencies at larger angles and thus an increasing single pass LPEF.

In Fig. 4, the variation of the single pass LPEF for different systems with respect to the refractive index is shown. As a comparative system to the cell and encapsulated case of the random pyramid structure and the 1000-nm grating, a Lambertian scatterer was used.

The refractive index dependent single pass LPEF shows a similar behavior for all the investigated coupling structures. In general, the function for all the systems is convex. In the small refractive index range, the changes in the refractive index result in small changes in the single pass LPEF. In the higher refractive index range, the changes in the refractive index lead to larger changes in the single pass LPEF. At $n=3.5$, the single pass LPEF for the Lambertian scatterer is much larger than that for the coupling structures. The single pass LPEF for both structures in the cell case is higher than that of the encapsulated case over the entire refractive index range. This is because of the lower refractive index contrast between the refractive index of the structure and the EVA encapsulation compared to the cell case, where the surrounding material is air. In the case of the pyramid system, the refractive power is weaker due to the lower refractive index

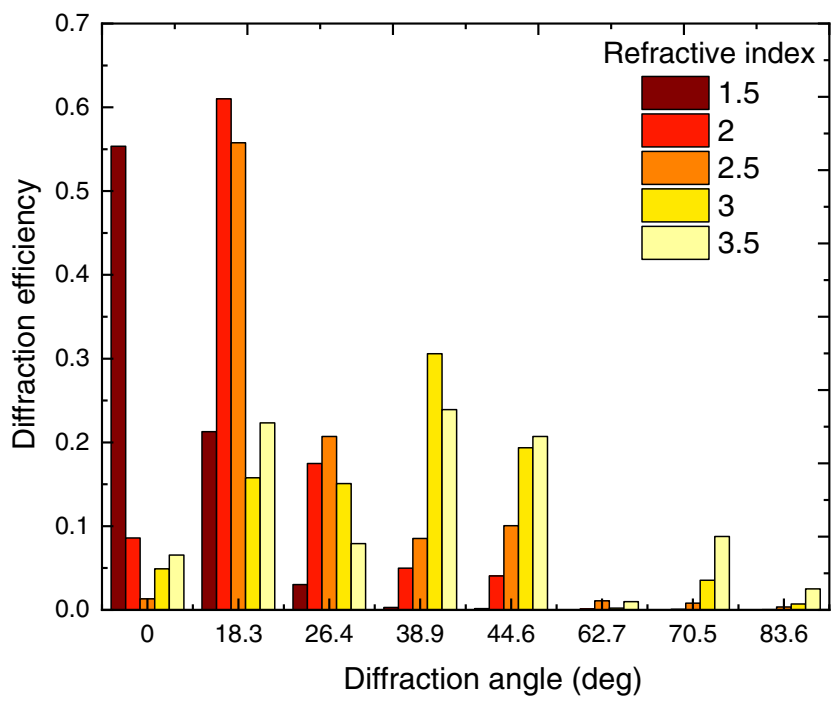

Fig. 3 The plot shows the diffraction efficiencies into the diffraction angles for five different refractive indices of the $1000-\mathrm{nm}$ grating at the wavelength $1100 \mathrm{~nm}$. It can easily be seen that structures with higher refractive index lead to higher diffraction efficiencies at larger angles. It is noteworthy that the angles on the $x$ axis are discrete diffraction angles that are determined by the grating equation. 


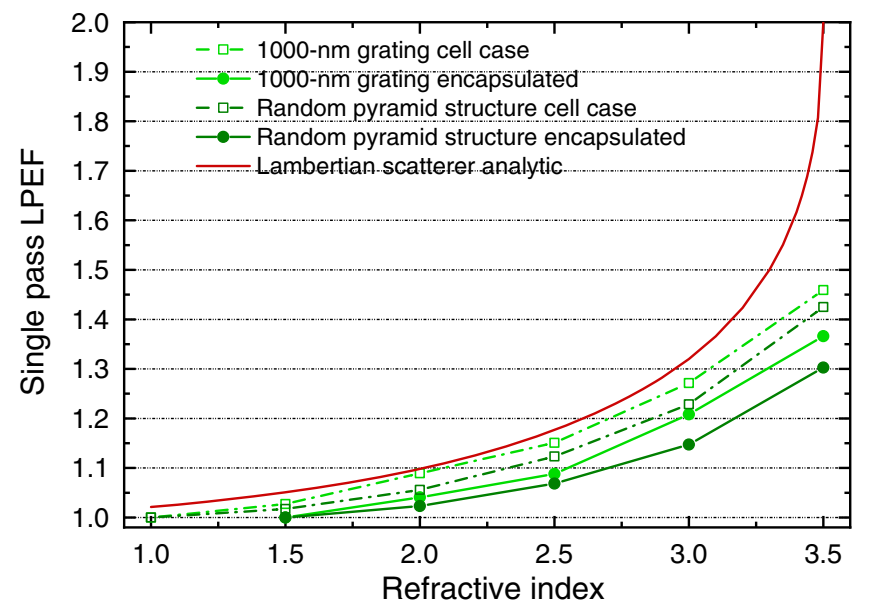

Fig. 4 Simulation results for the single pass LPEF for a random pyramid and a grating structure in comparison to a Lambertian scatterer.

contrast. Thus, the resulting angle in the absorber becomes smaller, while in the diffracting system the diffraction efficiencies of the larger angles decrease.

\section{Results: Complete System}

\subsection{System LPEF}

The system LPEF was calculated using the methods described in Sec. 2.7. The comparison of the single pass LPEF and the system LPEF is shown in Fig. 5.

All the system LPEFs are much higher than the single pass LPEF and the curve behavior changes. Looking, e.g., at the Lambertian scatterer the single pass LPEF doubles in the refractive index range from 1 to 3.5 , and the system LPEF increases in this range by a factor of 20. This clearly shows that the single pass LPEF can only provides a rough indication of the light-trapping behavior. In the system, the escape behavior becomes more important and has a dominant influence on light-trapping. This behavior is important for understanding the current density results. It is noteworthy that the Lambertian limit for light-trapping is often calculated using the simplified formula LPEF $=4 n^{2}$, which would be 49 in the case of $n=3.5$. However, this value is only achieved in the limit of absorption free material, which is not the case here. For a

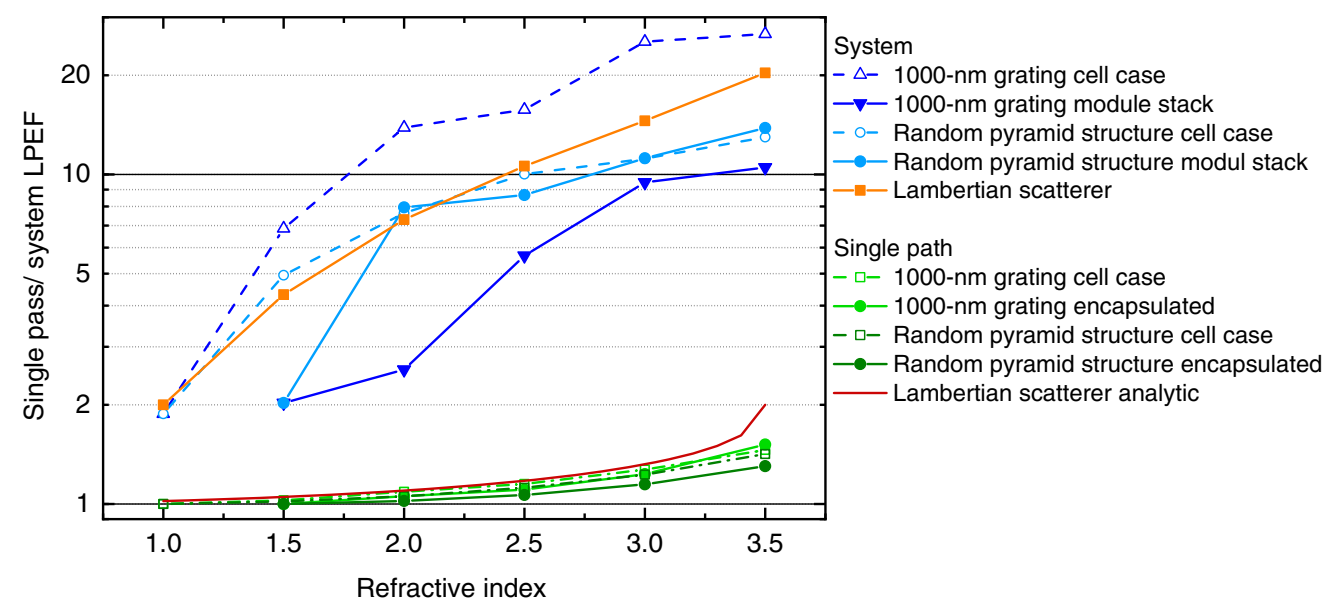

Fig. 5 The single pass and system LPEF of the different interfaces and of the Lambertian scatterer in a logarithmic plot. 
better estimation of the Lambertian limit accounting for the absorbing material, one can use the approximation shown in the work of Tiedje et al. [Ref. 19 and Eq. (7)]. In addition, it is noteworthy that the system LPEF was only calculated for normal light incidence. The extremely good behavior of the 1000-nm grating structure might not be maintained for different angles of incidence.

\subsection{Gain in Photo Current}

The method used to obtain the following results is described in Sec. 2.8. The results for the complete system simulation at the cell and module level are shown in Fig. 6. The results show the dependence of the current density gain (normalized to $100 \%$ front side transmittance) on the refractive index for the random pyramid structure, the 1000-nm grating, and the Lambertian scatterer. In the reference system, a silicon solar cell with a planar front side is used.

For the cell case, it can clearly be seen that the gain for the random pyramid structure and the Lambertian scatterer is close until the refractive index of 2.2. Then, the gain of the random pyramid structure increases slowly when compared to that of the Lambertian scatterer. The gain for the 1000-nm grating is over the complete refractive index range higher than that for the other two structures. This fits perfectly with the system LPEF results.

It is noteworthy that the current gain reaches its maximum when all the light is absorbed. The absorption gain due to path length enhancement follows the Lambert-Beer law, which is a "limited growth" $\boldsymbol{A} *(\mathbf{1}-\mathbf{e x p}(-\boldsymbol{b} * \boldsymbol{x}))$ function. As this limited growth dominates the current gain, the overall behavior shows limited growth. The results for the module case show that the current density gain for the random pyramid structure is significantly higher than that for the 1000-nm grating. This is in contrast to the results in the cell case. Therefore, in the module case, the amount of light that escapes from the random pyramid structure is less than that from the 1000-nm grating. The random pyramid structure shows a significant increase in the current density gain for small refractive indices from 1.5 to 2 . Here, the escape behavior of the random pyramid structure increases significantly. The random pyramid structure shows good light trapping behavior in the module simulation even if the absolute value of the current density gain is smaller than in the cell case.

If the results shown here are compared with the system LPEF results of the different coupling structures, they show similar trends. The 1000-nm grating has the lowest system LPEF, which results at the lower current density gain. The system LPEF of the random pyramid structure is higher than the 1000-nm grating, and the Lambertian scatterer had the highest system LPEF. However, the difference between all the systems was quite small. The trend in the current density gain with the variation of the refractive index are the same.

The refractive index variation was performed with artificial materials for this study. It is important to understand which materials can be used in a real device. While direct structuring

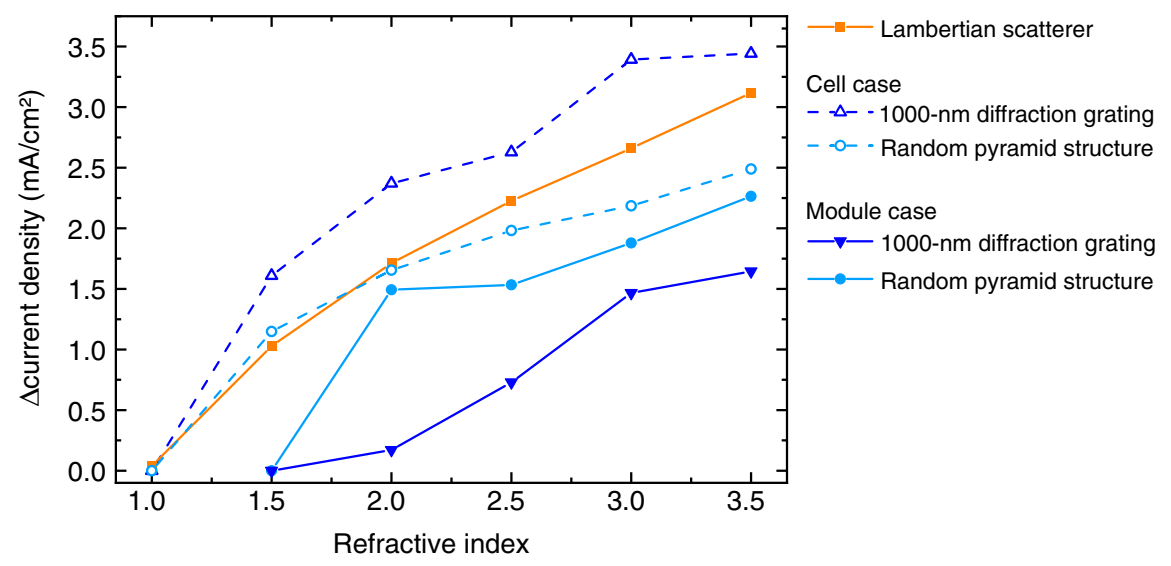

Fig. 6 Current density gain compared to a planar substrate for the random pyramid structure, the grating and the Lambertian scatterer in the cell and the module case. 
of the active silicon cell $(n \sim 3.5)$ is the state-of-the-art and, according to our study, allows for the highest LPEFs, sometimes this structuring is not possible or could be unwanted (e.g., in silicon-based tandem solar cells ${ }^{6,20}$ ). Thus, alternative ways of structuring have been investigated recently. ${ }^{7,9}$ Numerous low-refractive index materials $(n \sim 1.5)$ exist, and front side structures have already been realized with such materials, for example $\mathrm{PDMS}^{6}$ or polymethyl methacrylate (PMMA). Materials with refractive indices around 2 could be $\mathrm{SiN},{ }^{21} \mathrm{SiC},{ }^{22}$ or $\mathrm{TaO}^{23}$ However, for these materials $(n \leq 2)$, the gain is quite limited. Materials with a medium-high refractive index $(\sim 2.5)$ are more difficult to find. One prominent candidate is $\mathrm{TiO}_{2},{ }^{7,9}$ and it has been shown, that these structures can be used in solar cells. Materials with high refractive index that would allow for the highest current gains are usually strongly absorbing and thus not well suited for front side light-trapping structures, as the parasitic absorption loss usually will overcompensate the potential gain. The only relevant method for a high-refractive-index structure at the front side is the structuring of the active material. Thus, it can be concluded that in terms of light path enhancement, direct structuring of the active material is preferable, as long as this is possible.

Another option pointing beyond front side coupling is to realize light-trapping at the rear side of the device. In this way, structures in materials with high absorption coefficients or even metals can be realized such as amorphous silicon ${ }^{24}$ and silver. ${ }^{20}$ Therefore, a planar front side as well as an unstructured active cell can be maintained without limiting the light-trapping performance by the choice of non-absorptive materials.

\section{Conclusion and Outlook}

We showed that the refractive index of the coupling structure has a strong influence on the light path enhancement and light-trapping behavior. For the single pass LPEF, the random pyramid structure and the grating show similar results. Therefore, in this case, the influence of the coupling structure was found to be lower than that of the refractive index. By taking the complete system with multiple passes into account, the crossed diffraction grating on the cell level shows higher absorption which leads to higher current density gains. For an EVA encapsulated coupling structure, the current density gain is smaller than that for a coupling structure in air. The random pyramid structure was found to show high current density gains.

The results show that the well-known good light-trapping performance of random pyramidal textures also applies to pyramidal structures in other materials. Furthermore, the high potential of grating structures is demonstrated: If well designed, they can lead to a significant current density gain. This is mainly due to the excellent escape behavior. However, the strong difference between the cell and the module case show, that there is still room for optimization of the grating parameters. As the focus of this study was the influence of the refractive index, a global grating optimization is beyond the scope of this work.

In addition to the light-trapping optimization, it is very important to consider the transmission behavior at the front surface of the solar cell. This was not part of this simulation study and will be addressed in future work.

The present study offers a good basis for the selection and optimization of coupling structures for PV systems incorporating cells with planar front surfaces.

\section{Appendix}

\subsection{Calculation of Single Pass LPEF for the Random Pyramids}

In Fig. 7, a comparison between a planar substrate [Fig. 7(a)] and a pyramidal structured surface [Fig. 7(b)] is shown. The thickness of the planar substrate was effectively increased by the single pass LPEF from the pyramidal structured surface. Thus, both the systems would show the same absorption, even though the structured device is physically thinner.

With the help of a ray tracer from PV Lighthouse, the single pass LPEF of a system for the pyramidal structure with varying refractive index was calculated. As there is no direct access in PV Lighthouse to the resulting propagation angles in the material, a workaround was used that is 


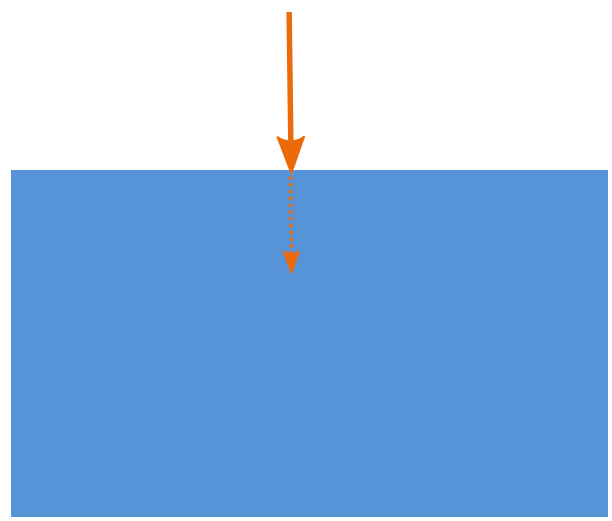

(a)

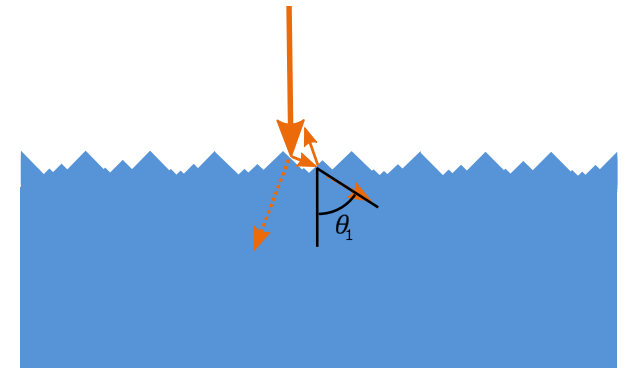

(b)

Fig. 7 (a) The single pass LPEF enlarged planar substrate that would generated the same absorption results like the (b) structured substrate.

based on the calculation of the absorptance in a very thin Si layer. The absorptance within such a layer can be written as

$$
\text { Absorptance }_{\text {total }}=\sum_{p} \eta_{p}\left(1-\exp \left(-\frac{\alpha d_{\text {substrate }}}{\cos \theta_{p}}\right)\right)
$$

where $\eta_{p}$ is the transmitted efficiency at a certain angle and $\alpha$ the absorption coefficient. As long as the absorption is very small, one can use the approximation:

$$
\text { Absorptance }=1-\exp \left(-\alpha d_{\text {substrate }}\right) \approx \alpha d_{\text {substrate }}
$$

and thus, rewrite Eq. (7) as

$$
\text { Absorptance }_{\text {total }} \approx \sum_{p} \frac{\eta_{p}}{\cos \theta_{p}} \alpha d_{\text {substrate }} \approx \alpha d_{\text {eff }} \quad \text { with } \quad d_{\text {eff }}=\sum_{p} \frac{\eta_{p} d_{\text {substrate }}}{\cos \theta_{p}}
$$

In other words, if we operate in this weakly absorbing regime, the system can be described by an effectively thicker substrate $\left(d_{\text {eff }}\right)$. The single pass LPEF by a structure can be calculated from the following:

$$
\mathrm{LPEF}_{\text {single }}=\frac{d_{\text {eff }}}{d_{\text {substrate }}}
$$

However, as the calculation is performed numerically, the thickness must be chosen such that the absorption is not too small (very close to 0) to prevent numerical issues. Thus, for the investigated wavelength of $1100 \mathrm{~nm}$, a silicon substrate with a thickness of $10 \mu \mathrm{m}$ was chosen for this calculation. The error was estimated by the comparison of the absorption for a light path with an angle of $\theta_{p}=60 \mathrm{deg}$. The absorption is 0.007 , and the error was found to be $0.3 \%$ relative, when compared to the exact value [Eq. (7)] of the approximation in Eq. (9). For lower propagation angles, this error even decreases and thus is negligible for the investigations in this study.

\subsection{Calculation of Single Pass LPEF for the 1000-nm Grating}

The result of the parameter variation for the 1000-nm grating single path LPEF is shown in Fig. 8 for the cell case and in Fig. 9 for the encapsulated case, where each plot represents a different refractive index of the structure. The different colors show the calculated single path LPEF with an identical scale for all plots. 

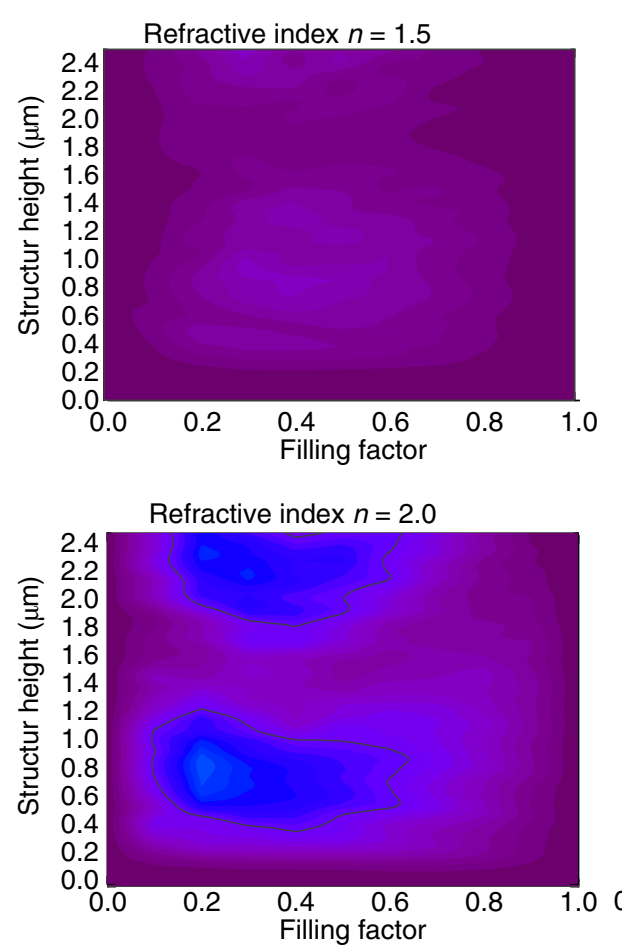

Refractive index $n=2.5$
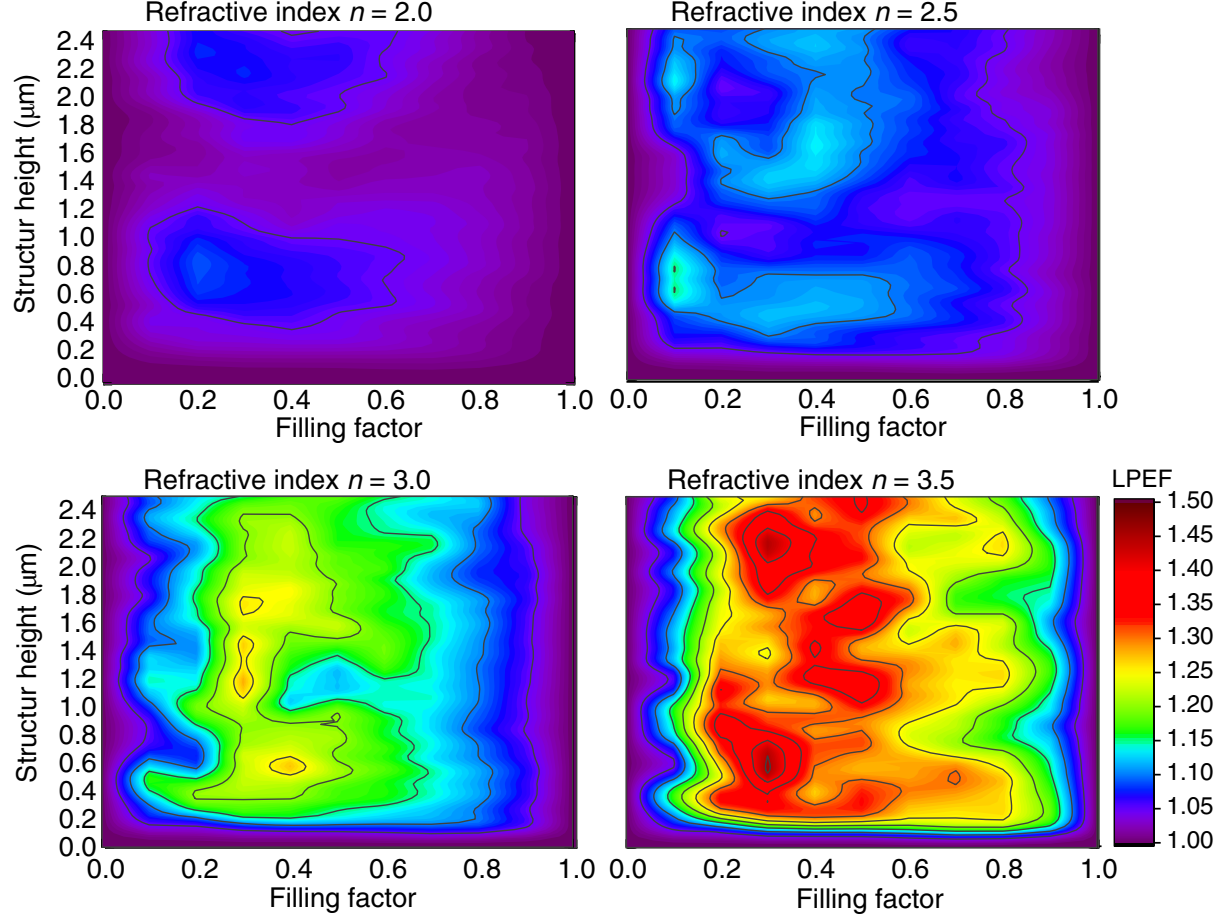

Fig. 8 This tableau shows the LPEF as a function of structure height and fill factor for a 1000-nm grating on air. Each contour plot represents a different refractive index from left to right and top to bottom in ascending order from 1.5 to 3.5 .

For each refractive index an optimum for the LPEF can be found in terms of the structure height and the filling factor. The maxima from all contour plots are presented in Table 1 for the cell case and for the grating structure in the encapsulated case in Table 2.

\subsection{Comments on the Calculation of the Single Pass LPEF for the Grating Structure}

The calculation of the single pass LPEF for the grating structure was performed by averaging over the four wavelengths $1000,1050,1100$, and $1150 \mathrm{~nm}$. It should be note that there will be differences if only four wavelengths are used to calculate the single pass LPEF. However, the differences are sufficiently small for comparing the influence of different refractive indices on the LPEF (compare Table 3). In particular, when looking at the final result of the system LPEF and the influence on the absorption within the system, it becomes clear that these deviations have a small influence on the final result, especially on the shown trends. Thus, with regard to the large simulation time, for the parameter variation described in Sec. 3, only four wavelengths 

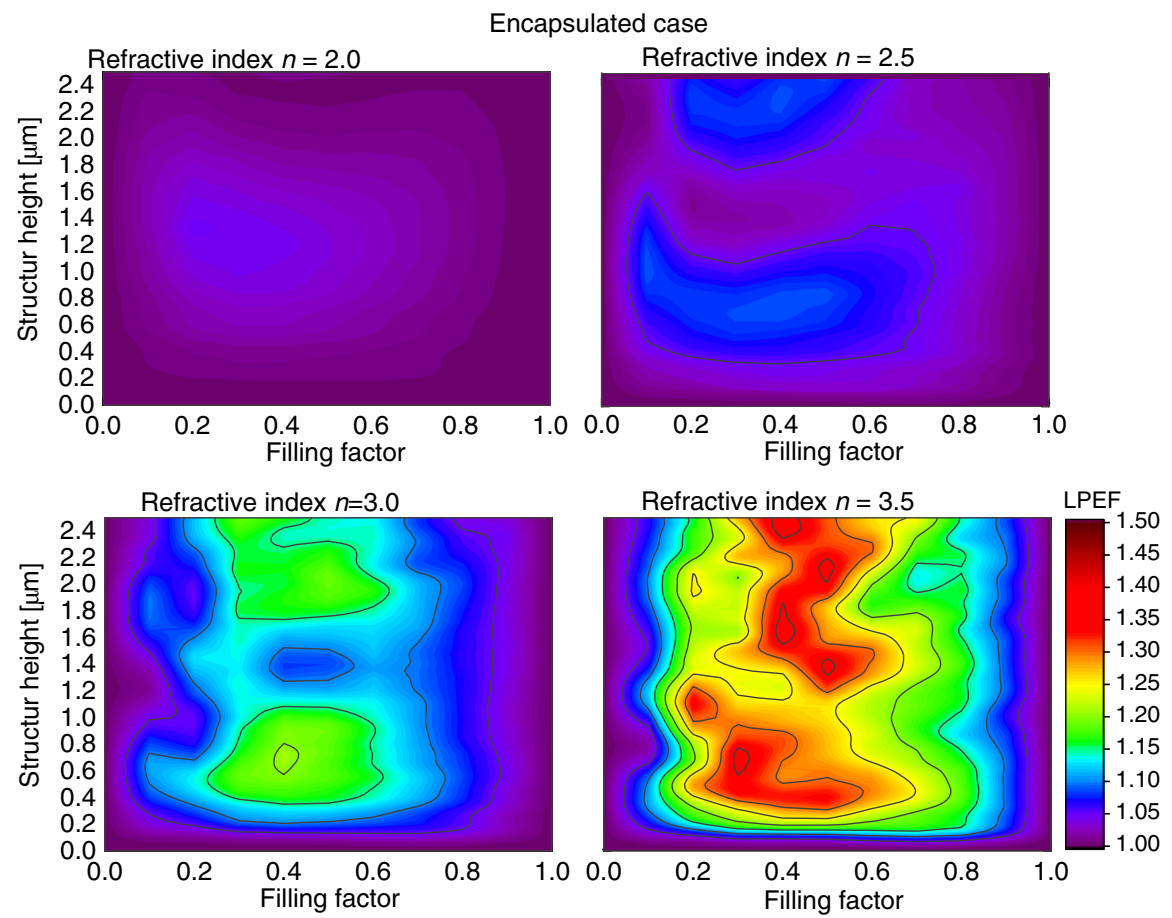

Fig. 9 This tableau shows the LPEF also as a function of structure height and filling factor for a $1000-n m$ grating in the module case, where the refractive index of the front side material is 1.5. Each contour plot represents another refractive index here beginning by 2 to 3.5 .

Table 1 Parameters of the maximal single path LPEF for each refractive index in the cell case.

\begin{tabular}{lccccccc}
\hline \hline $\begin{array}{l}\text { Refractive index } \\
\text { structure }\end{array}$ & $\begin{array}{c}\text { Structure } \\
\text { height }\end{array}$ & $\begin{array}{c}\text { Filling } \\
\text { factor }\end{array}$ & $\begin{array}{c}\text { LPEF (at } \\
1000 \mathrm{~nm})\end{array}$ & $\begin{array}{c}\text { LPEF (at } \\
1050 \mathrm{~nm})\end{array}$ & $\begin{array}{c}\text { LPEF (at } \\
1100 \mathrm{~nm})\end{array}$ & $\begin{array}{c}\text { LPEF (at } \\
1150 \mathrm{~nm})\end{array}$ & $\begin{array}{c}\text { LPEF } \\
\text { averaged }\end{array}$ \\
\hline 1.5 & 0.95 & 0.3 & 1.02 & 1.03943 & 1.0211 & 1.0244 & 1.03 \\
2 & 0.85 & 0.2 & 1.0805 & 1.0927 & 1.0928 & 1.091 & 1.09 \\
2.5 & 0.65 & 0.1 & 1.1209 & 1.1488 & 1.1775 & 1.1537 & 1.15 \\
3 & 0.575 & 0.4 & 1.2436 & 1.2584 & 1.3573 & 1.2312 & 1.27 \\
3.5 & 0.575 & 0.3 & 1.3269 & 1.3822 & 1.6003 & 1.5273 & 1.46 \\
\hline \hline
\end{tabular}

Table 2 Parameter of the maximal single path LPEF for each refractive index in the encapsulated case.

\begin{tabular}{lccccccc}
\hline \hline $\begin{array}{l}\text { Refractive index } \\
\text { structure }\end{array}$ & $\begin{array}{c}\text { Structure } \\
\text { height }\end{array}$ & $\begin{array}{c}\text { Filling } \\
\text { factor }\end{array}$ & $\begin{array}{c}\text { LPEF (at } \\
1000 \mathrm{~nm})\end{array}$ & $\begin{array}{c}\text { LPEF (at } \\
1050 \mathrm{~nm})\end{array}$ & $\begin{array}{c}\text { LPEF (at } \\
1100 \mathrm{~nm})\end{array}$ & $\begin{array}{c}\text { LPEF (at } \\
1150 \mathrm{~nm})\end{array}$ & $\begin{array}{c}\text { LPEF } \\
\text { averaged }\end{array}$ \\
\hline 2 & 1 & 0.2 & 1.0355 & 1.0358 & 1.0397 & 1.0353 & 1.04 \\
2.5 & 0.8 & 0.4 & 1.0854 & 1.0857 & 1.093 & 1.0905 & 1.09 \\
3 & 0.7 & 0.4 & 1.1905 & 1.1998 & 1.2321 & 1.2102 & 1.21 \\
3.5 & 0.7 & 0.3 & 1.3053 & 1.3754 & 1.4091 & 1.3722 & 1.37 \\
\hline \hline
\end{tabular}


Table 3 Structure parameter of the maximal single pass LPEF averaged over four wavelengths versus the LPEF averaged in the range from 1 to $1.2 \mu \mathrm{m}$ for each refractive index in the cell case.

\begin{tabular}{lcc}
\hline \hline Refractive index structure & LPEF (averaged four wavelength) & LPEF (average in range 1 to 1.2 $\mu \mathrm{m}$ ) \\
\hline 1.5 & 1.03 & 1.02 \\
2 & 1.09 & 1.09 \\
2.5 & 1.15 & 1.14 \\
3 & 1.27 & 1.26 \\
3.5 & 1.46 & 1.40 \\
\hline \hline
\end{tabular}

were considered. It is noteworthy that the system simulations for the chosen grating parameters were done with high resolution.

\section{Acknowledgments}

This work was partially funded by the German Federal Ministry of Economic Affairs and Energy within the project SOLGEL-PV (FKZ 0324151A). L. Stevens gratefully acknowledged scholarship support from the Deutsche Bundesstiftung Umwelt (DBU). The authors declare no conflicts of interest.

\section{References}

1. M. A. Green, High Efficiency Silicon Solar Cells, Trans Tech Publications, Kensington (1987).

2. P. Campbell and M. A. Green, "Light trapping properties of pyramidally textured surfaces," J. Appl. Phys. 62(1), 243 (1987).

3. A. Hauser et al., "A simplified process for isotropic texturing of mc-Si," in Proc. 3rd World Conf. Photovoltaic Energy Conversion, pp. 1447-1450 (2003).

4. S. Manzoor et al., "Improved light management in planar silicon and perovskite solar cells using PDMS scattering layer," Sol. Energy Mater. Sol. Cells 173, 59-65 (2017).

5. K.A. Bush et al., "Minimizing current and voltage losses to reach $25 \%$ efficient monolithic two-terminal perovskite-silicon tandem solar cells," ACS Energy Lett. 3(9), 2173-2180 (2018).

6. C. Yi et al., "Application of polydimethylsiloxane surface texturing on III-V//Si tandem achieving more than $2 \%$ absolute efficiency improvement," Opt. Express 28(3), 3895 (2020).

7. P. Spinelli et al., " $\mathrm{Al}_{2} \mathrm{O}_{3} / \mathrm{TiO}_{2}$ nano-pattern antireflection coating with ultralow surface recombination," Appl. Phys. Lett. 102(23), 233902 (2013).

8. P. Spinelli et al., "Effect of EVA encapsulation on antireflection properties of Mie nanoscatterers for c-Si solar cells," IEEE J. Photovoltaics 5(2), 559-564 (2015).

9. L. Stevens et al., "Broadband antireflection Mie scatterers revisited-a solar cell and module analysis," Opt. Express 27(8), A524 (2019).

10. K. Xu et al., "Micro optical sensors based on avalanching silicon light-emitting devices monolithically integrated on chips," Opt. Mater. Express 9(10), 3985-3997 (2019).

11. M. G. Moharam and T. K. Gaylord, "Rigorous coupled-wave analysis of metallic surfacerelief gratings," J. Opt. Soc. Am. A 3(11), 1780-1787 (1986).

12. K. McIntosh et al., "PV lighthouse: SunSolve ${ }^{\mathrm{TM}}$," https://www.pvlighthouse.com.au/ sunsolve.

13. N. Tucher et al., "Optical simulation of photovoltaic modules with multiple textured interfaces using the matrix-based formalism OPTOS," Opt. Express 24(14), A1083 (2016).

14. N. Tucher et al., "3D optical simulation formalism OPTOS for textured silicon solar cells," Opt. Express 23(24), A1720 (2015). 
15. M. A. Green, "Self-consistent optical parameters of intrinsic silicon at $300 \mathrm{~K}$ including temperature coefficients," Sol. Energy Mater. Sol. Cells 92(11), 1305-1310 (2008).

16. M. G. Moharam and T. K. Gaylord, "Diffraction analysis of dielectric surface-relief gratings," J. Opt. Soc. Am. 72(10), 1385-1392 (1982).

17. O. Höhn, N. Tucher, and B. Bläsi, "Impact of front side pyramid size on the light trapping performance of wafer based silicon solar cells and modules," in Proc. 44th IEEE Photovoltaic Specialists Conf. (2017).

18. IEC, "Photovoltaic devices-part 3. Measurement principles for terrestrial photovoltaic (PV) solar devices with reference spectral irradiance data," International Electrotechnical Commission (2008).

19. T. Tiedje et al., "Limiting efficiency of silicon solar cells," IEEE Trans. Electron Devices 31(5), 711-716 (1984).

20. R. Cariou et al., "III-V-on-silicon solar cells reaching 33\% photoconversion efficiency in two-terminal configuration," Nat. Energy 3(4), 326-333 (2018).

21. M. R. Vogt, "Development of physical models for the simulation of optical properties of solar cell modules," PhD Thesis, Gottfried Wilhelm Leibniz University, Hannover (2015).

22. B. Steinhauser et al., "Stacks of a-SiCx layers on planar and textured surfaces for the solar cell front side on both $\mathrm{n}^{+}$and $\mathrm{p}^{+}$-emitters," in Proc. 5th World Conf. Photovoltaic Energy Conversion, pp. 2250-2254 (2010).

23. T. J. Bright et al., "Infrared optical properties of amorphous and nanocrystalline $\mathrm{Ta}_{2} \mathrm{O}_{5}$ thin films," J. Appl. Phys. 114(8), 83515 (2013).

24. J. Eisenlohr et al., "Efficiency increase of crystalline silicon solar cells with nanoimprinted rear side gratings for enhanced light trapping," Sol. Energy Mater. Sol. Cells 155, 288-293 (2016).

Laura Stevens studied physics at the Technical University in Dortmund (Germany) and wrote her master's thesis at the Fraunhofer Institute for Solar Energy Systems (ISE) in the field of optical power converters. She received her master's degree in 2016 and started her $\mathrm{PhD}$ thesis on the topic "Patterned sol-gel-antireflective surfaces for increasing the light coupling in silicon photovoltaics" in 2017 at the Fraunhofer ISE and the Albert-Ludwigs-University of Freiburg. Her main research interests include optical modeling and the realization of micro- and nanostructured surfaces via nano-imprint lithography.

Biographies of the other authors are not available. 